COMUNICAÇÃO BREVE

\section{LA CRENOBALNEOTHERAPIE EN FRANCE: REVUE GENERALE}

\section{A CRENOBALNEOTERAPIA NA FRANÇA: VISÃO GERAL}

\section{RÉSUMÉ}

Après un bref historique, il s'agit de décrire le thermalisme français avec ses différentes sources et stations et les indications d'une cure thermale prises en charge par le système de soins.

\section{MOTS CLÉS}

Crénobalnéothérapie. France. Thermalisme. Santé Publique.

\section{ABSTRACT}

After a historical data, it is a description of French crenobalneotherapy, her springs and health-resorts, indications of spa-cure reimbursed by National health service.

\section{KEYWORD}

Crenobalneotherapy. France. Thermalism. Public Health.

\section{RESUMO}

Após uma breve história, descreve-sea balneoterapia francesa a partir das diferentes fontes e estações termais e as respectivas indicações de tratamentos financiadas pelo sistema público de saúde.

\section{PALAVRAS-CHAVE}

Balneoterapia. França. Termalismo. Saúde Pública.

\section{INTRODUCTION}

Lutilisation traditionnelle de l'eau thermale est très ancienne en France puisque le plus vieux site a été découvert près de Vézelay et date d'environ 2000 ans avant Jésus-Christ. Le thermalisme s'est ensuite développé avec la conquête romaine. Ceux ci ont fondé une partie des stations françaises qui sont encore en activité aujourd'hui comme Aix-les-Bains, Vals-les-Bains, Bourbonne-les-Bains...

Historiquement, celles-ci étaient peu médicalisées jusqu’à la moitié du $18^{\text {ème }}$ siècle $^{1}$. Les installations étaient sommaires voire absentes et un observateur rapporte que « les baigneurs étaient exposés aux

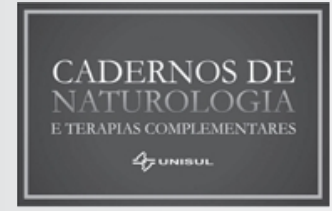

Romain Forestier ${ }^{1}$

Waïner Tabone ${ }^{2}$

Michel Palmer ${ }^{3}$

Pascale Jeambrun ${ }^{4}$

Jean-Baptiste Chareyras ${ }^{4}$

Didier Guerrero ${ }^{4}$

Régine Fabry ${ }^{5}$

Alain Françon ${ }^{1}$

$1 \mathrm{MD}$, centre de recherche rhumatologique et thermal Aix-LesBains, 15, avenue Charles de Gaulle, 73100 Aix-Les-Bains, France. Société Française de Médecine Thermale.

2 Association française pour la recherche thermale.1, rue Cels, 75014 Paris, France

3 MD Syndicat national des médecins thermaux, 3, place des Thermes, 73100 Aix-Les-Bains, France

$4 \mathrm{MD}$, Société Française de Médecine Thermale,

5 Senior Lecturer (Maître de Conférences) University d'Auvergne CLERMONT I, 28, place Henry DUNANT, BP 38. F-63001 CLERMONT FERRAND CEDEX

C ORRES P ONDENTE

Pascale Jeambrun Rua Newton Romanelli, 150 37705-032 - Jardim Hortênsias, Poços de Caldas/MG.

E - M A I L pascale.jeambrun@wanadoo.fr Recebido: 03/03/2014 Aprovado: 21/07/2014 
intempéries ». À partir de cette période, le thermalisme médical se développe, appuyé sur les inventaires et analyses des sources thermales réalisés par les scientifiques, souvent à la demande des monarques ; à partir de la fin du dix-huitième siècle les cures thermales sont régulièrement fréquentées par les soldats revenant de campagnes militaires.

$\mathrm{Au}$ milieu du dix-neuvième siècle, le développement des chemins de fer favorise l'apparition et le développement de stations thermales un peu partout en
Europe et particulièrement en France. Elles sont alors fréquentées par la clientèle, notamment anglaiseprivilégiée et fortunée. Cette activité, mi mondaine, mi thérapeutique se poursuivra jusqu’à la fin de la «belle époque " avec la deuxième guerre mondiale.

Lannée 1947, marque le début du remboursement des cures thermales par la Sécurité sociale et lessor du thermalisme social et médicalisé dans le pays.

Un exemple de fréquentation est donné dans la figure 1 pour la station d'Aix-les-Bains (Savoie France).

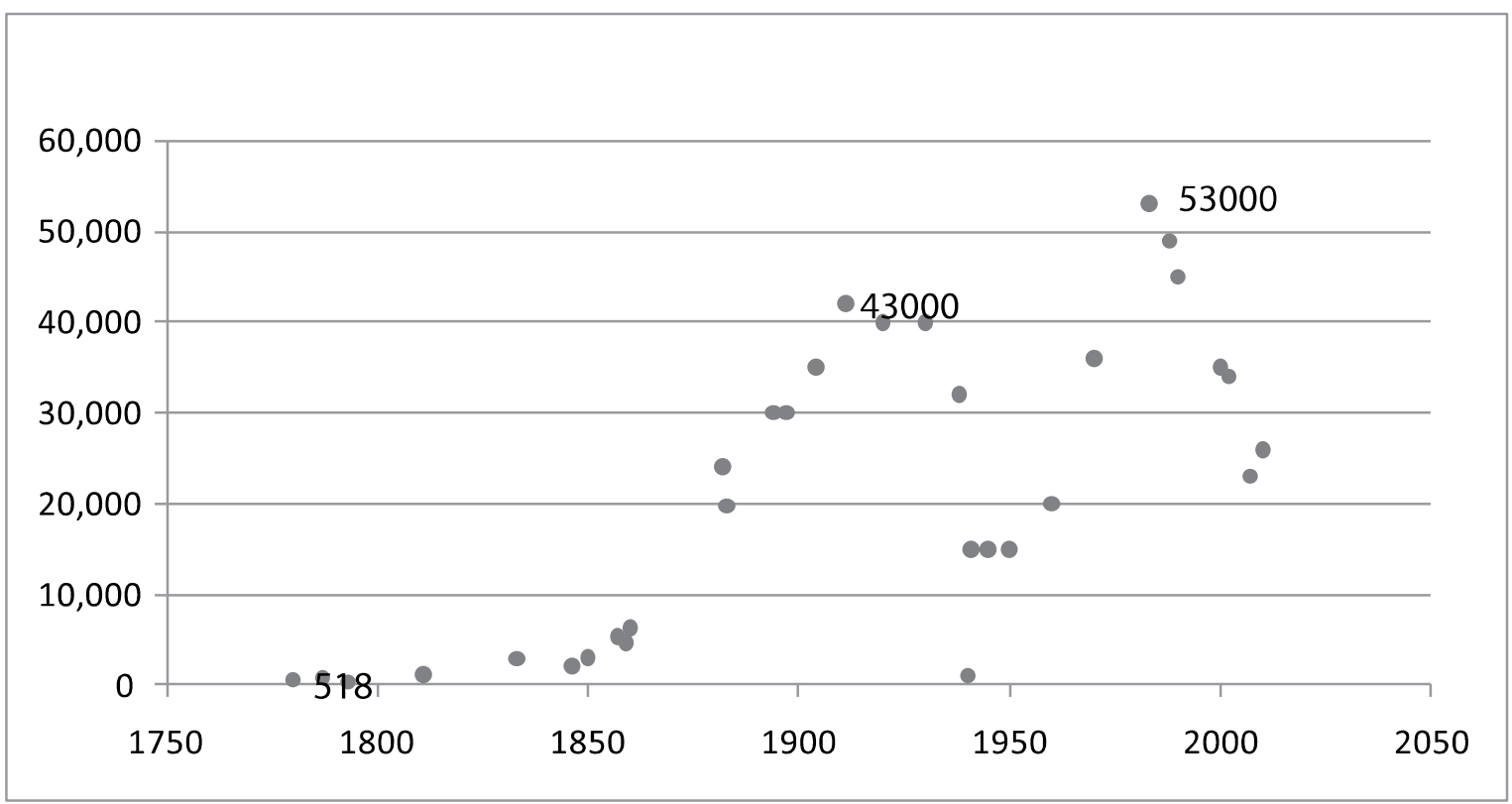

Figure 1: nombre de patients/an à Aix-les-Bains de 1780 à 2010.

\section{NOMBRE DE STATIONS THERMALES, STATISTIQUES DE FREQUENTATION}

Les stations sont classées en fonction de leur orientation thérapeutique (tableau 1). Lors d'une cure, un patient peut en suivre une ou deux, selon les possibilités de la station et les pathologies dont il souffre.

Tableau 1: Fréquentation des différentes spécialités thérapeutiques thermales en France de 1991 à 2010

\begin{tabular}{lrrrr}
\hline & $\mathbf{1 9 9 1}$ & $\mathbf{\%}$ & $\mathbf{2 0 1 0}$ & $\mathbf{\%}$ \\
\hline Rhumatologie & 302583 & $55,72 \%$ & 370195 & $75,10 \%$ \\
\hline voies respiratoires & 114703 & $21,12 \%$ & 44417 & $9,01 \%$ \\
\hline voies urinaires et digestives & 43016 & $7,92 \%$ & 26533 & $5,38 \%$ \\
\hline Phlébologie & 38444 & $7,08 \%$ & 17702 & $3,59 \%$ \\
\hline Dermatologie & 14024 & $2,58 \%$ & 12980 & $2,63 \%$ \\
\hline Pathologie cardiaque et artérielle & 14624 & $2,69 \%$ & 6250 & $1,27 \%$ \\
\hline Psychiatrie & 9412 & $1,73 \%$ & 9040 & $1,83 \%$ \\
\hline Neurologie & 3668 & $0,68 \%$ & 5304 & $1,08 \%$ \\
\hline Troubles du développement de l'enfant & 651 & $0,12 \%$ & 60 & $0,011 \%$ \\
\hline Gynécologie & 1446 & $0,27 \%$ & 274 & $0,06 \%$ \\
\hline Stomatologie & 439 & $0,08 \%$ & 184 & $0,01 \%$ \\
\hline Total & 543010 & $100,00 \%$ & 292939 & $100,00 \%$ \\
\hline
\end{tabular}




\section{GEOGRAPHIE ET CLASSIFICATION DES EAUX MINERALES ET THERMALES}

En France, on classe les eaux thermales en fonction de leur température, leur origine géologique et leur composition chimique ${ }^{2}$.

\section{Géographie et répartition des principales stations thermales en France}

La France compte 105 stations thermales réparties sur tout le territoire (figure 1). Elles sont, pour la plupart, situées dans ou à proximité des massifs montagneux (figure 2).

\section{Les sources des Vosges}

Les principales stations sont Niederbrohn et Morsbrohn sur le versant alsacien Sur l'autre versant Lorrain, en allant vers le sud et louest on trouve Amnéville, Plombières, Bains les Bains, Luxeuil, puis Bourbonne, Contrexéville et Vittel

\section{Les sources des Alpes}

Dans la zone du Briançonnais et du chablais on trouve les sources chaudes de Monetier les Bains, Brides les Bains et Salins, avec une eau chlorosulfatée mixte.

La zone des massifs cristallins externes comporte des émergences sulfureuses ou chlorosulfureuses avec les stations de la Léchère, Allevard les BainsetSaint Gervais-les-Bains.

La zone subalpine fournit des eaux sulfureuses tièdes et peu minéralisées de Digne-Les-Bains, Uriage, Marlioz et Challes-les-Eaux

Dans la zone molassique, la plus externe par rapport à l'arc alpin, on trouve deux types de sources :

» les eaux chaudes sulfureuses d'Aix-les-Bains,

»les eaux non sulfureuses et peu minéralisées

de Thonon et Evian-les-Bains.

\section{Les sources des Pyrénées}

Dans la zone axiale, on trouve des sources sulfurées sodiques et chaudes de Bagnères-de-Luchon, Barèges et Cauterets.

Dans la zone nord pyrénéenne, on trouve des minéralisations chlorosulfatées ou sulfatées calciques de Bagnères-de-Bigorre et Capvern.

Dans la zone pré-pyrénéenne on ne trouve que des sources froides à minéralisation chlorurée sodique : Salies-de-Béarn, Salies-du-Salat.
La dernière zone des alluvions quaternaires donne des eaux chloro-sulfatées de Dax et Barbotan.

\section{Les sources du massif central}

Elles comprennent Vichy, La Bourboule, Le MontDore, Royat, Châtel-Guyon, Saint Nectaire, Vals et Bourbon-Lancy. Il s'agit essentiellement d'eaux bicarbonatées

\section{Les sources du Jura}

Il s'agit essentiellement de sources salées :Salins et Lons-le-Saunier, ou de sources peu minéralisées : Divonne-les-Bains.

\section{Les autres sources}

On en trouve

»dans le bassin parisien : Enghien-les-Bains ;

»en Normandie Forges; dans le Nord :

Saint-Amand-les-Eaux;

»en Normandie: Bagnoles-de-L'Orne;

»dans le Poitou: La Roche-Posay;

»en Charentes Maritimes : Rochefort, Saujon.

\section{TEMPÉRATURE}

Les eaux froides font moins de $20^{\circ} \mathrm{C}$, elles sont appelées les eaux hypothermales : de $21^{\circ}$ à $35^{\circ} \mathrm{C}$, les eaux mesothermales : de $35^{\circ}$ à $45^{\circ} \mathrm{C}$ et les eaux hyperthermales : plus de $45^{\circ} \mathrm{C}$ à l'émergence.

Cette classification historique ne reflète plus les températures utilisées depuis que les stations s'approvisionnent sur des forages parfois très profonds dont la température peut être différente. Par exemple à Aix-les-Bains, les sources historiques "d'Alun " et de "Souffre " qui émergeaient à $45^{\circ}$ et $55^{\circ}$ sont remplacée par un forage « Reine Hortense " à $1100 \mathrm{~m}$ de profondeur qui émerge à $38^{\circ}$ et un forage "Chevalley " à $2200 \mathrm{~m}$ de profondeur qui émerge à $71^{\circ}$.

\section{ORIGINE GÉOLOGIQUE}

On distingue les eaux "vadoses » qui résultent de l'infiltration des eaux de pluie et les eaux " géothermiques " qui résultent de la libération de molécules d'eau à partir des réactions chimiques se produisant dans le manteau terrestre. 


\section{COMPOSITION CHIMIQUE}

Elle est fortement influencée par les minéraux traversés lors de lémergence. On classe les eaux en fonction des ions dominants dans leur composition. En superposition de ces classifications légales, les chimistes ont proposé un certain nombre de classifications. Nous en proposons l'une d'elles.

\section{Eaux bicarbonatées}

Les plus courantes sont

» Bicarbonatées sodiques

»Bicarbonatées calciques

»Bicarbonatées mixtes

Elles sont traditionnellement recommandées dans le traitement des affections digestives mais les stations les utilisent également en rhumatologie

Eaux Sulfureuses

Eaux Sulfatées

»Sulfatées calciques

»Sulfatées chlorurées

»Eaux radioactives (à haute teneur en radon)

Eaux Sulfurées

»Sulfurées calciques

»Sulfurées mixtes

»Et sulfurées sodiques

\section{Eaux chlorurées}

Chlorurées sodiques faibles moyennes et fortes

Eaux à composition spéciale

Eaux ferrugineuses

Caractérisées par une forte teneur en fer, mais elles sont généralement associées à d'autres minéraux comme les bicarbonates et les sulfates

Comme les précédentes, elles sont traditionnellement recommandées dans le traitement des affections digestives mais sont également utilisées en dermatologie et en rhumatologie

Eaux arsenicales

Eaux riches en cuivre
PRINCIPALES INDICATIONS DU TRAITEMENT THERMAL, METHODES DE TRAITEMENT DANS LES CENTRES THERMAUX

En France, les cures thermales remboursées par la Sécurité sociale sont systématiquement délivrées sur une période de trois semaines. Elles sont semi standardisées et comportent 72 soins prescrits par le médecin thermal au début de la cure après examen du patient.

Les indications traditionnelles sont très nombreuses dans les traités ${ }^{3}$ mais les progrès de la médecine moderne ont réduit les indications.

Les pratiques thermales sont variables d'une station à l'autre mais peuvent être regroupées sous différentes catégories. Elles avaient été recensées dans les différents établissements français par le syndicat des médecins thermaux en $20044^{4}$.

\section{EN RHUMATOLOGIE}

\section{Indications principales}

Les principales indications sont les pathologies dégénératives: lombalgies, cervicalgies chroniques et arthrose $e^{5}$. Nous avons quelques statistiques personnelles non publiées qui détaillent les principaux diagnostics des patients débutant une cure thermale

Tableau 3: diagnostic des patients débutant une cure thermal en pratique de ville (données personnelles sur 245 patients consécutifs vus en avril 2011).

\begin{tabular}{lr}
\hline DIAGNOSTICS & N \\
\hline Pathologies dégénératives & \\
\hline arthrose généralisée (3 localisation ou plus) & 99 \\
\hline Arthrose rachidienne isolée & 27 \\
\hline Scoliose & 3 \\
\hline arthrose de la hanche isolée & 3 \\
\hline arthrose du genou isolée & 5 \\
\hline hyperostose vertébrale & 13 \\
\hline Pathologie de la coiffe des rotateurs & 7 \\
\hline Arthropathies microcristallines & 15 \\
\hline Chondrocalcinose & 1 \\
\hline Hémochromatose & \\
\hline Pathologie inflammatoire &
\end{tabular}




\begin{tabular}{lr}
\hline DIAGNOSTICS & N \\
\hline Polyarthrite Rhumatoïde & 11 \\
\hline Spondylarthrite ankylosante & 7 \\
\hline Arthrite psoriasique & 2 \\
\hline $\begin{array}{l}\text { Maladie de Horton et Pseudopolyarthrite } \\
\text { rhizomélique }\end{array}$ & 6 \\
\hline Sclérodermie & 1 \\
\hline Syndrome de Sjögren primitif & 1 \\
\hline Lupus érythémateux disséminé & 1 \\
\hline Autres & 1 \\
\hline Ostéoporose fracturaire & 6 \\
\hline Algodystrophie & 31 \\
\hline Fibromyalgie & $\mathbf{2 4 5}$ \\
\hline $\begin{array}{l}\text { Absence de confirmation radiologique } \\
\text { ou biologique }\end{array}$ & \\
\hline Total &
\end{tabular}

sur la base des essais thérapeutiques publiés nous considérons que le traitement thermal est un traitement indiqué dans la lombalgie chronique ${ }^{6}$, les cervicalgies ${ }^{7-8}$ l'arthrose du genou ${ }^{9}$ l'arthrose de la main ${ }^{10}$, la polyarthrite rhumatoïde ${ }^{11}$, la fibromyalgie $^{12-13}$ la spondylarthrite ankylosante ${ }^{14,27}$.

\section{Méthodes de traitement}

Les massages, les applications de boue, les bains, les douches et les piscines sont les plus utilisées.

Les bains sont réalisés en baignoire par séances de 10 ou 20 minutes. La température des bains est réglée spécifiquement pour chaque patient, en moyenne de $34^{\circ}$ à $38^{\circ}$. Il peut s'agir de bains simples, de séances de douches sous-marines administrées par un technicien thermal ou de bains avec hydrojets.

Les massages sont proposés par séances de 10 minutes. Ils sont réalisés sous une douche d'eau thermale à $38^{\circ}$. Ils sont délivrés par un personnel qualifié (kinésithérapeute).

Les boues sont proposées dans toutes les stations rhumatologiques par séances de 10,15 ou 20 minutes. Il peut s'agir de cataplasmes, enrobés dans un tissu ou d'applications directes. La température séchelonne de 43 à $50^{\circ}$ selon les stations. Elle peut aussi être délivrée sous forme de bains de limon thermal qui est en fait un bain en eau thermale additionnée d'argile. Dans ce cas, les températures sont plus basses : de 29 à $38^{\circ}$.

Les piscines peuvent être des séances libres, associées à des jets d'hydromassages ou comporter des mouvements supervisés par un personnel qualifié (kinésithérapeutes). La température de l'eau est de 32 à $36^{\circ}$ et la durée du soin peut être de 10 à 30 minutes. Le nombre de patients par groupe de rééducation est variable selon les stations, de 6 à 30 . Les mouvements comportent généralement des exercices de délordose pour le rachis lombaire, de décoaptation pour les épaules et du travail en amplitude et en étirement pour les autres articulations.

Les douches peuvent être automatiques ou délivrées spécifiquement sur les zones douloureuses par les techniciens thermaux. Elles sont généralement à une température de $38^{\circ}$ et durent 3 à 6 minutes.

Il y a également des traitements plus spécifiques dans certaines stations. On signale le Berthollet d'Aix-les-Bains : Brouillard d'eau thermale appliqué pendant $10^{\prime}$ sur les localisations douloureuses à une température de $38^{\circ}$. À Dax on réalise des douches térébenthinées : douches thermales avec ajout d'essence de térébenthine. À Royat on pratiquait des injections d'eau bicarbonatées péri articulaires mais cette technique est peu à peu abandonnée. On utilise enfin les bains de gaz sec qui durent $10^{\prime}$ à $38^{\circ}$.

\section{EN OTO-RHINO-LARYNGOLOGIE ET PNEUMOLOGIE}

\section{Principales indications}

Les cures ORL sont principalement prescrites pour les infections chroniques ou récidivantes : les rhino-pharyngites récidivantes, les rhinites et les rhino-sinusites chroniques, les otites aiguës récidivantes et les otites séromuqueuses, l’amygdalite chronique et les angines récidivantes, les laryngites sous-glottiques.

Parfois, elles sont également prescrites pour une pathologie allergique : la rhinite allergique, les rhino-sinusites allergiques et les toux spasmodiques. 
En pneumologie, l'indication principale est l'asthme de l'adulte et de l'enfant, mais on l'utilise aussi dans les bronchiolites de l'enfant, la bronchite chronique et la dilatation des bronches.

\section{Méthodes de traitement}

Les techniques de détersion lavage sont les gargarismes, les bains nasaux, les irrigations nasales, la douche pharyngée et les pulvérisations nasales et pharyngées. Elles utilisent la projection d'eau thermale directement sur les muqueuses ORL par le patient lui-même.

Certaines stations utilisent des techniques d'hydrothérapie générales similaires à celles décrites plus haut : bains en baignoire, bains en piscine, douches au jet.

La rééducation respiratoire est réalisée par des kinésithérapeutes diplômés. Elle peut se faire en séances individuelles ou collectives de 15 à 20 minutes.Elle comporte des techniques de travail diaphragmatique, de gymnastique narinaire et faciale, de relaxation musculaire et de gymnastique posturale.

Les inhalations collectives en ambiance chaude (35 à $38^{\circ}$ ) saturée d'humidité. Parfois, l'atmosphère est naturellement chargée d'humidité, d'autre fois on ajoute un nébulisateur ou un aérosol dans la pièce. Il existe également des inhalations individuelles avec les mêmes techniques. Les aérosols individuels diffusent à l'aide d'un masque des particules de 2 à 20 microns obtenues par nébulisation (aérosols soniques). Les séances durent de 10 à 20 minutes.

Trois types de soins sont délivrés par les médecins thermaux eux mêmes :

»L'insufflation tubaire, on introduit une sonde jusqu'à l'entrée de la trompe d'eustache et on délivre un jet de gaz à pression progressivement croissante.

»La méthode de déplacement de Proëtz réalise une aspiration-dépression des sinus de la face. Elle nécessite une décongestion préalable de la muqueuse nasale et sinusienne.

»La douche pharyngienne est un jet, dispensé sous un réglage filiforme ou dispersé, sous contrôle de la vue, à l'aide d'un pistolet pharyngien

\section{EN PATHOLOGIE ARTERIELLE}

\section{Principales indications}

- Artériopathie Oblitérante des Membres Inférieurs,

- Les phénomènes de Raynaud primaires et secondaires.

Le $\mathrm{CO}_{2}$ naturel qui accompagne les eaux thermo-minérales Chloro-Bicarbonatées Sodiques est utilisé à létablissement thermal de Royat-Chamalières/France, mais aussi dans 43 établissements allemands et dans de nombreux pays européens Autriche, République Tchèque, etc.....où le $\mathrm{CO}_{2}$ est incorporé par voie percutanée.

Pour nous, seules les eaux carbo-gazeuses dont la teneur en $\mathrm{CO}_{2}$ libre est $\geq 1 \mathrm{~g} / \mathrm{kg}$ peuvent avoir un effet thérapeutique car la diffusion transcutanée est conditionnée par un gradient $\geq 50 \mathrm{mmHg}$. L'effet vasoactif est maximum pour les modalités d'application suivantes: immersion sous-sternale ; $\left[\mathrm{CO}_{2}\right]=1800 \mathrm{mg} / \mathrm{kg}^{5}$; température du bain $34-35^{\circ} \mathrm{C}$; durée du bain 20-30 minutes ; fréquence du bain 1824 jours. Dans ces conditions, les effets physio-pharmacologiques mis en évidence par des essais contrôlés : augmentation locale des débits sanguins ; ouverture des capillaires fonctionnellement fermés, dilatation des segments pré-capillaires ; l'augmentation de la PCO2 entraine une libération accrue d'oxygène à partir de la forme combinée oxyhémoglobine (effet Bohr) ; déformabilité globulaire augmentée, modification du seuil des thermorécepteurset effet antiseptique. Les effets de la diffusion transcutanée $\mathrm{du} \mathrm{CO}_{2}$, vasodilatation artériolaire et libération de loxygène tissulaire ${ }^{1}$.

\section{Méthodes de traitement}

À Royat-Chamalières le traitement est dispensé selon les recommandations précédentes pendant 18jours consécutifs :

\section{Pour les syndromes de Raynaud}

1 - Bain des avant-bras pendant 10minutes ( $\theta$ $=34,5^{\circ} \mathrm{C}$ ) suivi par 20 minutes de gaz sec.

2 - Immersion générale en bain carbo-gazeux $\left[\mathrm{CO}_{2}=1,6 \mathrm{~g} / \mathrm{kg}\right]$ durant 20minutes 
3 - Immersion locale des avant-bras en atmosphère gazeuse.

\section{Pour l'artériopathie oblitérante des membres inférieurs}

1 - Immersion générale, sous-sternale, en bain carbo-gazeux $\left[\mathrm{CO}_{2}=1,6 \mathrm{~g} / \mathrm{kg}\right]$ durant $20 \mathrm{mi}$ nutes, $\left(\theta=34,5^{\circ} \mathrm{C}\right)^{6}$.

2 - Bain de gaz sec sur peau préalablement humidifiée ${ }^{6}$.

3 - Couloir de marche à contre-courant comprenant le parcours de 2 anneaux l'un central, de distance de 23mètres avec une profondeur de $40 \mathrm{~cm}$; l'autre externe, de distance de 33mètres et d'une profondeur de $60 \mathrm{~cm}$. La séance est de 10 minutes. 4 - Bain carbo-gazeux local de jambe alterné avec une douche locale spécifique pendant 10 minutes.

5 - Des insufflations sous-cutanées de $\mathrm{CO}_{2}$ naturel peuvent être réalisées au niveau des zones sténoses ; elles sont injectées en sous-cutané par les médecins thermaux à l'aide d'une aiguille.

On utilise parfois les cures de boisson : un à 2 verres d'eau minérale carbogazeuse par jour.

\section{Dans les pathologies digestives, les maladies de l'appareil urinaire et les maladies métaboliques}

\section{Principales indications}

"Maladies de l'appareil digestif : Troubles fonctionnels intestinaux, la maladie diverticulaire, les séquelles de maladies parasitaires intestinales, les formes légères des maladies inflammatoires $d u$ tube digestif (Crohn, rectocolite hémorragique).

»Maladies métaboliques : surpoids et obésité, troubles du métabolisme lipidique et diabète.

»Lithiase urinaire, infections urinaires basses récidivantes, les prostatites chroniques ou récidivantes, les cystalgies non infectieuses.

\section{Méthodes de traitement}

\section{Méthodes internes}

La cure de boisson est l'élément principal. Elle est prescrite de façon personnalisée par le médecin pour chaque patient. Généralement on utilise de pe- tites quantités d'eau entre 18 et $36^{\circ}$ ( 2 prises de 30 à $70 \mathrm{gr}$ avant les repas). Elle a un effet régulateur sur le transit intestinal et sur les sécrétions digestives et un effet antiseptique sur la flore intestinale.

L'Entéroclyse ou goutte à goutte intestinal : consiste en l'instillation lente (20 à 40 minutes) d'eau thermale à $37^{\circ}$. Généralement il s'agit de $200 \mathrm{~g}$ d'eau thermale mais les quantités peuvent être réduites ou augmentées jusqu'à $500 \mathrm{~g}$. Elle a un effet cicatrisant, calmant, anti-inflammatoire et un effet de rééducation ano-rectale.

La douche intestinale consiste en un lavement simple à l'eau thermale portée à $37^{\circ}$. On administre 1 à 3 litres d'eau en 5 à 10 minutes.

\section{Méthodes externes}

On utilise des bains et des douches déjà décrits plus hauts, des cataplasmes de boue en application abdominale, des massages abdominaux par des kinésithérapeutes.

On réalise également des pulvérisations d'eau thermale anale et périnéale.

\section{EN DERMATOLOGIE}

\section{Principales indications}

Leczéma et la dermatite atopique représente $60 \%$ des indications, le psoriasis cutané environ 30\%. On utilise parfois le thermalisme pour les séquelles de brûlures dès la sortie de l'hôpital.

\section{Méthodes de traitement}

Les douches filiformes sont souvent considérées comme le soin essentiel face aux processus cicatriciels pathologiques. Elles utilisent un ou des jets très fins (2 à 30 dixième de millimètres) et à forte pression (2 à 15 bar). Cette douche est administrée par le médecin thermal pendant des séances de 3 à 10 minutes selon les cas et permet une détersion des lésions cutanées. Selon les situations, la distance avec la peau sera plus ou moins importante selon que l'on recherchera un effet de détersion ou de massage énergique. Ce soin, potentiellement douloureux etparfois très pénible pour le patient, nécessite une grande expérience de la part du praticien. 
Les bains généraux durent de 5 à 30 minutes. Il peut s'agir parfois de bains simples, de bains avec douches sous-marines (dans ce cas elles sont appliquées par un technicien thermal) ou de bains avec hydrojets déjà décrits plus haut.

On utilise aussi des pulvérisations simples d'eau thermale pendant 3 à 10 minutes et des compresses deau thermale. On réalise enfin des massages manuels sous douche d'eau thermale et parfois des cures de boisson.

\section{EN PHLEBOLOGIE}

\section{Principales indications}

L'insuffisance veineuse chronique sous ses différentes formes : œdème chronique, dermite de stase, hypodermites et ulcères, varices et séquelles de phlébites.

\section{Méthodes de traitement}

Des massages ascendants sont destinés à faciliter la résorption des œdèmes. Ils sont réalisés par des kinésithérapeutes pendant des périodes de 10 minutes.

Bains et douches, généralement à des températures plus basses qu'en rhumatologie, plutôt vers $32-33^{\circ}$. On met l'accent sur la profondeur des baignoires avec minimum $50 \mathrm{~cm}$ d'eau pour obtenir une pression hydrostatique suffisante. On conseille une mobilisation active des jambes pendant le bain.

Les piscines ont l'avantage de pouvoir être plus profondes, la température conseillée est de 32 à $33^{\circ}$ pendant une durée de 10 à 15 minutes. Le couloir de marche est une piscine de déambulation qui est arpentée par les patients pendant 10 à 20 minutes.

Lors d'une double indication, on utilise différentes sortes de douches ou des applications de boues sur les membres inférieurs, dont la température est plus basse que pour les soins rhumatologiques, 36 à $39^{\circ}$ maximum.

Kneippthérapie : alternance de jets chauds et froids sur les jambes par séances de 10 minutes.

\section{AFFECTION PSYCHOSOMATIQUES}

\section{Principales indications}

La meilleure indication est l'anxiété généralisée ${ }^{15}$, mais on utilise aussi la cure thermale dans les so- matisations, les troubles du sommeil, les troubles dépressifs réactionnels et pour le sevrage vis-à-vis de la dépendance ou de l'abus de substances psychotropes.

\section{Méthodes de traitement}

La douche médicale est une des spécificités du traitement thermal des affections psychiatriques. Elle est délivrée par le psychiatre lui-même pendant une durée de 3 minutes.

L'enveloppement hydrothérapique consiste à envelopper le patient dans un drap imbibé d'eau thermale.

Les bains en piscine ou en baignoires, les douches, les massages sont similaires à ceux décrits plus haut.

\section{COUVERTURE SOCIALE DES CURE THERMALES}

Depuis 1947, la Sécurité sociale rembourse les cures thermales à la condition quelles soient prescrites par un médecin et qu'elles soient administrées par un établissement thermal régulièrement conventionné.

La convention nationale définit, en concertation avec les établissements thermaux, le contenu du forfait qui est pris en charge. Elleprécise le nombre, la durée des soins et la qualification du personnel requis pour les délivrer. Le strict respect de ce cahier des charges conditionne le remboursement par la protection sociale. Dans ce cadre une cure thermale dure obligatoirement 3 semaines (18 jours de soins). Le curiste assuré social bénéficie du remboursement des soins thermaux et des honoraires du médecin thermal qui suit le déroulement de la cure. Lorsque le revenu annuel des patients est inférieur à $14000 €$, ils peuvent également recevoir un remboursement partiel du transport, sur la base du prix du billet de train et de l'hébergement dans la station : environ $150 €$. Chaque année 500000 patients bénéficient d'une cure thermale dans l'une des 105 stations conventionnées, pour un coût total remboursé par la Sécurité sociale d'environ 200 millions euros $(<0,5 \%$ des dépenses de santé). 


\section{ÉDUCATION ET SYSTEME DE QUALIFICATION DES PRATICIENS ET DES TECHNICIENS THERMAUX}

\section{Formation}

\section{Formation des médecins thermaux}

Lexercice de la médecine thermale est libre mais il est conseillé de passer un diplôme pour exercer en station thermale.Celui-ci est délivré dans les universités de Montpellier, Grenoble, Clermont-Ferrand et Nancy. La médecine thermale est classée dans les médecines à exercice particulier. Il y a environ 1000 médecins exerçant la médecine thermale en France.

\section{Formation du personnel des établissements thermaux}

Les kinésithérapeutes sont formés dans les écoles de kinésithérapie. Il n’y a pas de formation spécifique pour le thermalisme qui applique essentiellement des méthodes de massage et de rééducation en piscine.

Autres membres du personnel : les agents des soins, qui délivrent les divers soins de balnéothérapie (bains, douches, applications de boues ou de vapeur, aérosolthérapie) en exécution de la prescription rédigée par le médecin thermal. Ces personnels bénéficient de formatons initiales délivrées par des instituts spécialisés et de formation continue.

\section{SOCIETE SAVANTE}

La société savante desmédecins thermaux français est la Société Française de Médecine Thermale, anciennement appelée Société Française d'Hydrologie et de Climatologie Médicales. Elle a été fondée en 1853. Elle a environ 300 adhérents qui sont des médecins thermaux, des praticiens non thermaux et des enseignants intéressés par la médecine thermale. Elle édite un journal en langue française avec abstract en anglais: la Presse thermale et climatique [http://www.lapressethermale.org]

\section{LA RECHERCHE THERMALE EN FRANCE}

\section{Les centres de recherche thermaux}

Il existe 4 centres de recherche spécifiquement dédiés à la médecine thermale en France.
»L'Institut du thermalisme de Dax qui dépend de l'université de Bordeaux

»Le Centre de recherche universitaire de la Léchère (CRULL) qui dépend de l'université de Grenoble

"L'Institut de Recherche cardio-vasculaire de Royat (IRCV) qui dépend de l'université de Clermont-Ferrand

»Le Centre de recherche rhumatologique et thermal d'Aix-les-Bains qui est une institution de bénévoles non universitaires.

\section{L'association française pour la recherche thermale}

Elle a été créée en 2004 par le Conseil national des établissements thermaux, organisme national qui regroupe l'ensemble des établissements thermaux français. Elle est financée par les établissements thermaux et des fonds publics.

Un comité scientifique fait des appels à projets et sélectionne les sujets les plus susceptibles de contribuer à l'évaluation de l'utilité de la médecine thermale. Elle a permis de réaliser des études d'efficacité à plus grande échelle et de motiver différentes équipes universitaires pour bâtir des projets. Les deux premiers sont déjà publiés dans le domaine de l'arthrose du genou ${ }^{9}$ et l'anxiété généralisée ${ }^{15}$. D'autres, sont en cours de publication ou d'analyse dans la pathologie chronique de la coiffe des rotateurs, les séquelles de phlébites, la perte de poids.

Depuis quelques années, l'AFRETH subventionne également des recherches destinées à développer de nouveaux champs de compétences pour la médecine thermale.

\section{Principales publications françaises}

\section{Rhumatologie}

À Nancy Guillemin puis Constant ont publié sur les lombalgies chroniques en 1994,1995 et 1998;16-18 puis en 2001 sur les pathologies rhumatologiques sous la direction du professeur Boulangé. Ils ont montré une amélioration de la gêne fonctionnelle, de la douleur et une diminution de la consommation médicamenteuse

À Aix-Les-Bains, Graber-Duvernay a publié sur la lombalgie chronique ${ }^{19}$ puis l'arthrose de la main ${ }^{10}$. 
Forestier a publié sur l'arthrose des membres inférieurs $^{20}$ la cervicalgie chronique $\mathrm{e}^{7-8} \mathrm{et}$ la gonarthrose $\mathrm{e}^{9}$ avec, là encore, une amélioration significative de la douleur et du handicap fonctionnel.

Nguyen a publié sous la direction du Pr Dougados une étude sur la lombalgie, la gonarthrose et la coxarthrose $^{21}$

\section{Cour et vaisseaux}

À Royat, Schmidt ${ }^{22}$ a montré l'effet de la cure sur le phénomène de Raynaud. Il a comparé 2 durées d'application du CO2 (9 vs 18 jours) et mesuré ses effets vasomoteurs dans les Syndromes de Raynaud ${ }^{8}$. Les résultats montrent l'efficacité du bain carbo-gazeux dans le groupe traité pendant 18 jours sur les paramètres microcirculatoires et clinique qui se traduit par une meilleure réponse à un test au froid en période hivernale

R Fabry a publié sur l'artériopathie des membres inférieurs $^{23,28}$.L'application percutanée de 18jours de

\section{FONTE DE FINANCIAMENTO}

\author{
Nenhuma
}

\section{CONFLITO DE INTERESSES}

Declara não haver

\section{REFERENCES}

1. Boyer M. Le thermalisme dans le grand sud est de la France. Presses universitaires de Grenoble. Grenoble, 2005.

2. Urbain P. Les sources thermo-minérales en France :étude géologique In Précis de pharmacologie et de thérapeutique hydrominérale. Lexpansion scientifique française. Paris, 1964.

3. Justin-Besançon L. Prescription des cures thermales, in Précis de pharmacologie et de thérapeutique hydrominérale. L'expansion scientifique française. Paris, 1964.

4. Palmer M. Guide des bonnes pratiques thermales.Press Therm Climat 2004,141:101-43.

5. Boulangé M., Guenot C., Fournier B., Gueguen R. Prévalence de la maladie rhumatismale et incidence du suivi des cures thermales chez les consultants âgés de 55 à 64 ans en France. Press Therm Climat1999,136(3):149-56.

6. Delcambre B., Jeantet M., Laversin S., Auberge T., Crenn O., Forestier R., et al. Diagnostic, prise en charge et suivi des malades atteints de lombalgie chronique. Recommandations de bonne pratique clinique, Haute Autorité de Santé. Décembre, 2000.

7. Forestier R., Francon A., Saint Arroman F., Bertolino C., GraberDuvernay B., Guillemot A., et al.Y-a-t-il un effet des soins thermaux et des champs électromagnétiques pulsés sur la cervicalgie chronique ? Essai clinique randomisé. Deuxième partie : approche médico-économique. Ann Readapt Med Phys 2007,50(30):148-53.
CO2 augmente immédiatement la distance totale de marche (critère principal) de $+66 \%$,quelle que soit la valeur de la marche initiale. Les résultats de cet essai clinique corroborent ceux rapportés antérieurement par Hartmann ${ }^{28}$, la CO2-thérapie apporte un véritable Service Médical Renduchez le claudicant parunemeilleure adaptation à la marche qui se maintient à un an.

Carpentier (Grenoble, la Léchère) a montré l'effet de la boue thermale sur la microcirculation cutanée ${ }^{24}$ Plus récemment, il a montré le bénéfice clinique d'une cure sur l'insuffisance veineuse chronique $^{25-26,30}$.

\section{Neurologie et psychiatrie}

À Toulouse, Befel-Couron, sous la direction du professeur Montastruc, a montré une amélioration de la qualité de vie dans la maladie de parkinson ${ }^{28}$. Dubois a montré que le traitement thermal était supérieur à la paroxétine dans l’anxiété généralisée ${ }^{15}$
8. Forestier R., Francon A., Saint-Arromand F., Bertolino C., Guillemot A., Graber-Duvernay B., et al. A-t-il un effet des soins thermaux et des champs électromagnétiques pulsés sur la cervicalgie chronique ? Essai clinique randomisé. Première partie : évaluation clinique. Ann Readapt Med Phys 2007,50(30):140-7.

9. Forestier R., Desfour H., Tessier Jm., Françon A., Foote Am., Genty C., et al. Spa therapy in the treatment of knee osteoarthritis: a large randomised multicentre trial.Ann Rheum Dis. 2010 Apr,69(4):660-5.

10. Graber-Duvernay B., Forestier R., Françon A. Efficacité du Berthollet d'Aix-les- Bains sur les manifestations fonctionnelles de l'arthrose des mains. Essai thérapeutique contrôlé. Rhumatologie 1997,49(4):151-6.

11. Mayoux Benamou A., Andre-Vert J., Scemama O., Guillez P., Celerier S., Combe B., et al.Polyarthrite rhumatoide : Prise en charge non pharmacologique Aspects thérapeutiques et socio-professionnels hors chirurgie. Haute Autorité de santé. Recommandation pour la pratique clinique 2007.[12] Zijlstra Tr., Van De Laar Ma., Bernelot Moens Hj., Taal E., Zakraoui L., Raskers Jj. Spa treatment for fibromyalgia syndrome: a combination of thalassotherapy, exercice and patient education improves symptoms and quality of life. Rheumatology 2005,44(4):539-46.

12. Zijlstra Tr., Braakman-Jansen Lm., Taal E, Rasker Jj., Van De Laar Ma.,Coste. Effectiveness of spa treatment for fibromyalgia: general health improvement is not for free. Rheumatology (oxford) 2007,46(9):1454-9. 
13. Van Tubergen A., Landewé R., Van Der Heijde D., Hidding A., Wolter N., Ascher M., et al. Combined spa-exercise therapy is effective in patient with ankylosing spondylitis: a randomized controlled trial. Arthritis Care Res. 2001,45:430-38.

14. Dubois O., Salamon R., Germain C., Poirier Mf., Vaugeois C. Banwarth B., et al., Olié JP. Balneotherapy versus paroxetine in the treatment of generalized anxiety disorder. Complement Ther Med. 2010 Feb,18(1):1-7.

15. Guillemin F., Constant F., Collin Jf., Boulangé M. Short and longterm effects of Spa therapy in chronic low back pain. British J Rheum 1994,33(2):148-151.

16. Constant F., Collin J F., Guillemin F., Boulangé M. Effectiveness of spa therapy in chronic low back pain: a randomised clinical trial.J Rheumatol 1995,22:1415-20.

17. Constant F., Collin J F., Guillemin F., Boulangé M. Use of spa therapy to improve the quality of life of chronic low back pain patients. Med Care 1998,36(9): 1409-14.

18. Llorca G., Graber-Duvernay B., Larbre Jp., Briançon D., Duplan B. Evaluation de l'efficacité de la cure thermale à Aix-les-Bains sur la lombalgie chronique de l'adulte. Etude contrôlée prospective.Rev Rhum 1995;764.

19. Forestier R. Magnitude and duration of the effects of two spa therapy courses on knee and hip osteoarthritis: an open prospective study in 51 consecutive patients.Joint Bone Spine. 2000,67(4):296-304.

20. Nguyen M., Revel M., Dougados M. Prolonged effects of 3 weeks therapy in a spa resort on lumbar spine, knee and hip osteoarthritis: Follow up after 6 months. A randomised controlled trial. Br J Rheumatol 1997,36:77-81.

21. Schmidt J., Monnet P., Normand B., Fabry R. Microcirculatory and clinical effects of serial percutaneous application of carbon dioxide in primary and secondary Raynaud's phenomenon. Vasa. 2005,34(2):93-100.
22. Fabry R., Monnet P., Schmidt J., Lusson J-R., Carpentier P-H., Baguet J-C., et al. Clinical and microcirculatory effects of transcutaneous $\mathrm{CO} 2$ therapy in intermittent claudication. Randomized double-blind clinical trial with a parallel design. Vasa 2009,38:213-224.

23. Poensin D., Carpentier Ph., Féchoz C., Gasparini S. Effects of mud pack treatment on skin microcirculation.Joint Bone Spine. 2003 Sep,70(5):367-70.

24. Carpentier Ph., Féchoz C., Poensin D., Satger B.Influence of spray application of La Léchère mineral water on the cutaneous microcirculation in the lower limbs in healthy subjects.J Mal Vasc. 2002 Oct,27(4):211-3.

25. Carpentier Ph., Satger B. Evaluation of balneotherapy associated with patient education in patients with advanced chronic venous insufficiency: A randomized controlled trial in the spa resort of La Léchère. J Vasc Surg 2008

26. Bannwarth B., Dernis Labous E., Streit G., Blanchard P., Boutry N., Brebant M., et coll. Diagnostic, prise en charge thérapeutique et suivi des spondylarthrites.Haute Autorité de santé. Recommandation pour la pratique clinique 2007.

27. Brefel-Courbon C., Desboeuf K., Thalamas C., Galitzky M., Senard Jm., Rascol O., Montastruc JL. Clinical and economic analysis of spa therapy in Parkinson's disease.Mov Disord. 2003 May,18(5):578-84.

28. Hartmann B., Bassenge E., Hartmann M. Effects of serial percutaneous application of carbon dioxide in intermittent claudication: results of a controlled trial. Angiology 1997,48:957-63.

29. Carpentier Ph., Blaise S., Satger B., Genty C., Rolland C., Roques C., et al. A multicenter randomized controlled trial evaluating balneotherapy in patients with advanced chronic venous insufficiency. J Vasc Surg. 2014 Feb,59(2):447-454 